\title{
Guided-wave signal processing using chirplet matching pursuits and mode correlation for structural health monitoring
}

\author{
Ajay Raghavan and Carlos E S Cesnik ${ }^{1}$ \\ Department of Aerospace Engineering, University of Michigan, 1320 Beal Avenue, \\ Ann Arbor, MI 48109, USA \\ E-mail: cesnik@umich.edu
}

Received 10 June 2006, in final form 1 November 2006

Published 30 January 2007

Online at stacks.iop.org/SMS/16/355

\begin{abstract}
Signal processing algorithms for guided wave pulse echo-based structural health monitoring (SHM) must be capable of isolating individual reflections from defects in the structure, if any, which could be overlapping and multimodal. In addition, they should be able to estimate the time-frequency centers, the modes and individual energies of the reflections, which would be used to locate and characterize defects. Finally, they should be computationally efficient and amenable to automated processing. This work addresses these issues with a new algorithm employing chirplet matching pursuits followed by a mode correlation check for single point sensors. Its theoretical advantages over conventional time-frequency representations for SHM are elaborated. Results from numerical simulations and experiments in isotropic plate structures are presented, which show the capability of the proposed algorithm. Finally, the issue of in-plane triangulation is discussed and experimental work done to explore this issue is presented.
\end{abstract}

(Some figures in this article are in colour only in the electronic version)

\section{Introduction}

\subsection{Motivation}

In recent years, there has been an emerging need for damage prognosis systems in aerospace structures. Such systems would automatically provide the user with near-real-time information about the structure's condition and its ability to continue service. In addition, they should also be able to give estimates of the remaining service life, thereby allowing repairs to be scheduled accordingly. Their importance has particularly come to the fore in the light of aerospace accidents such as the Aloha Airlines fuselage separation [1], the disintegration of the Space Shuttle Columbia during re-entry [2] and, more recently, the Chalk's Ocean Airways crash [3]. While both the aircraft incidents involved aging aircraft with fatigue-induced

\footnotetext{
1 Author to whom any correspondence should be addressed.
}

damage, the Space Shuttle disaster was due to its inability to detect damage to the wing by falling debris. In all cases, the presence of damage prognosis systems could have saved several lives. Apart from the obvious life safety benefits, the monetary and labor savings benefits of such systems would be very significant. More details on the motivational drivers for damage prognosis in civil, mechanical and aerospace structures can be found in Farrar et al [4], where it is aptly described as a 'grand challenge' engineering problem for the 21 st century.

\subsection{Structural health monitoring and guided wave approaches}

Structural health monitoring (SHM) is the diagnostic part that directly supports the damage prognosis. It usually consists of a structurally integrated network of transducers and processors that use a tested algorithm on the acquired data from the 

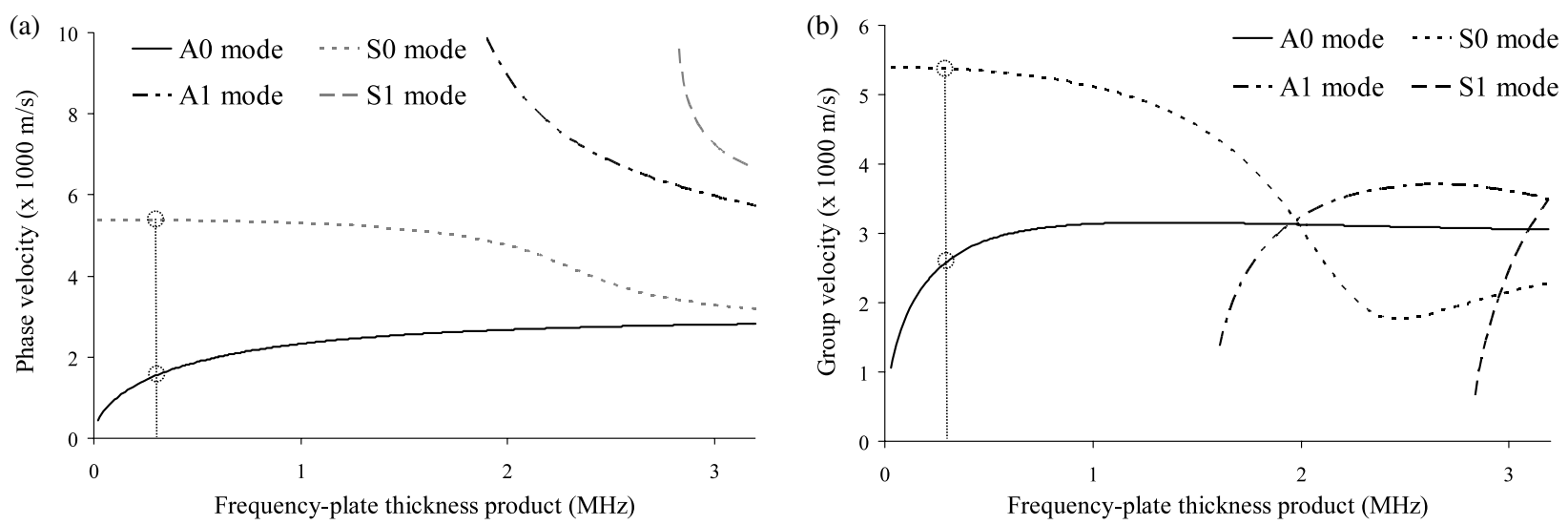

Figure 1. Dispersion curves for Lamb modes in an isotropic aluminum plate structure: (a) phase velocity and (b) group velocity. The circles mark the excitation center frequency for the FEM simulations in section 1.3.

transducers to evaluate the structure's condition. The SHM component would decide whether damage is present in the structure and, if so, furnish information about its location, severity and type. This would enable the damage prognosis system to make an intelligent estimate about the service potential of the structure. Among various technologies under investigation for SHM, there are guided wave (GW)-based approaches. These essentially involve exciting the structure with high frequency stress waves and processing the difference in structural response with respect to a baseline signal for the pristine condition using a tested algorithm to detect damage and characterize it, if present. Details on the basics of GW SHM and a brief comparison with other SHM methods are presented in another work by the authors [5]. The idea of GW SHM derives from GW non-destructive testing (NDT), which is now a well-established industrial technology. However, GW testing for SHM and NDT differs in two key aspects. The first one is that the mass and space penalty associated with transducers for NDT make them unsuitable for permanently mounting onto aerospace structures. Therefore, typically surface-bonded/embedded piezoelectric wafer transducers are used. The other significant difference is that the signal processing and inference about the presence of damage and its characteristics should be done automatically in near-realtime by a processor and not by a technician's judgment during offline maintenance as in NDT. This present paper addresses the signal processing aspect of GW SHM. In particular, drawbacks of conventional approaches to this aspect are highlighted and a new approach that overcomes these is presented and validated.

GWs can be defined as stress waves forced to follow a path defined by the material boundaries of the structure. For example, when a beam is excited at high frequency, stress waves travel in the beam along its axis away from the excitation source, i.e. the beam 'guides' the waves along its axis. Typical aerospace structures are composed of several beam, plate and shell substructures, each of which can act as waveguides, thereby making them attractive application areas for GW SHM. Typically, more than one mode is possible in a waveguide at any frequency. Furthermore, each mode has a unique dispersion curve, which represents the relation between phase velocity (denoted $c_{\mathrm{p}}$ ) and frequency. In isotropic plate structures at any excitation frequency, at least two modes are possible (see figure 1(a)). Another important characteristic is the group velocity (denoted $c_{\mathrm{g}}$, see figure $1(\mathrm{~b})$ ), defined as the derivative of the angular frequency with respect to the wavenumber. For an isotropic medium, it gives a very good approximation to the speed of the peak of the modulation envelope of a narrow frequency bandwidth pulse. This approximation improves in accuracy as the pulse moves further away from the source or if the GW mode becomes less dispersive. Further details on the fundamentals of GW propagation can be found in textbooks such as the ones by Miklowitz [6] or Graff [7].

\subsection{Issues in $G W$ signal processing for $S H M$}

Signal processing is a crucial aspect in any GW-based SHM algorithm. The objective is to extract information from the sensed signal to decide if damage has developed in the structure and, if so, characterize it in terms of location. Information about damage type and severity is also desirable from the signal for further prognosis. However, classifying and quantifying damage usually requires some pattern recognition algorithm which uses the output from the signal processing. The present work addresses signal processing, but not pattern recognition. To assess the issues involved in signal processing for GW SHM, results from a couple of illustrative finite element method (FEM) simulations are presented in this section. Consider a 2D aluminum plate structure, modeled using a finite element mesh of $2 \mathrm{D}$ plane strain elements as shown in figure 2(a) (the structure is infinitely wide normal to the plane of the paper). In the first simulation, a notch is present. It is $0.5 \mathrm{~mm}$ deep and $0.25 \mathrm{~mm}$ across, at a distance of $7.5 \mathrm{~cm}$ from the plate center. There are surface-bonded thin piezoelectric wafer actuators on each free surface at the center. The actuators are modeled as causing shear traction along their free edges, which has been found to be an effective model in previous work by the authors [8]. The actuators are excited symmetrically with a 2.5-cycle Hann-windowed sinusoidal toneburst with center frequency of $275 \mathrm{kHz}$. This frequency is highlighted in figure 1. Even though only the $S_{0}$ mode is excited in this case, when it interacts with a defect, all possible modes are scattered from the defect. At $275 \mathrm{kHz}$, three GW modes (the 
Guided-wave signal processing using chirplet matching pursuits and mode correlation for SHM

(a)

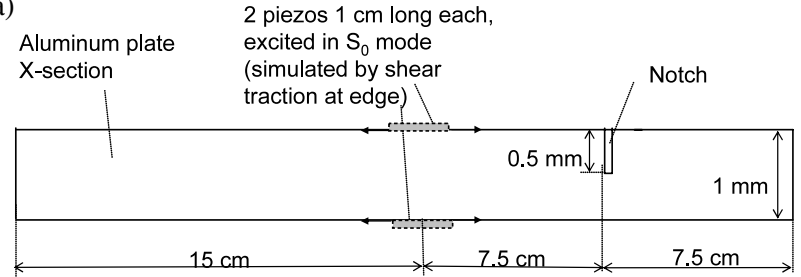

(d)

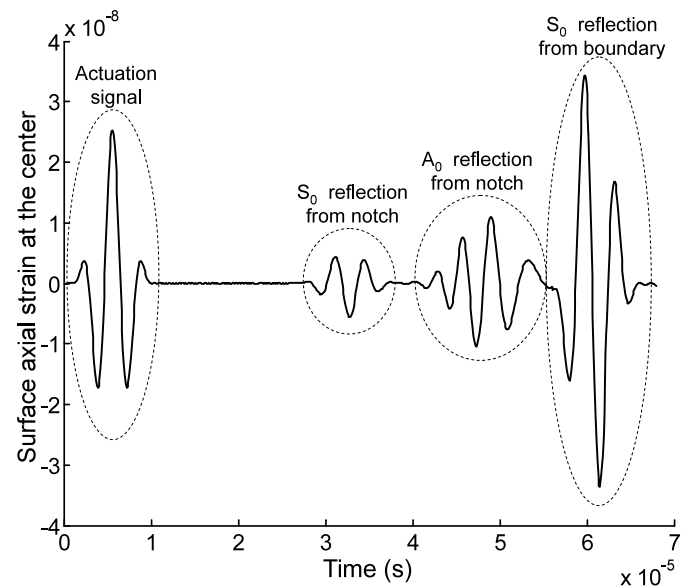

(b)

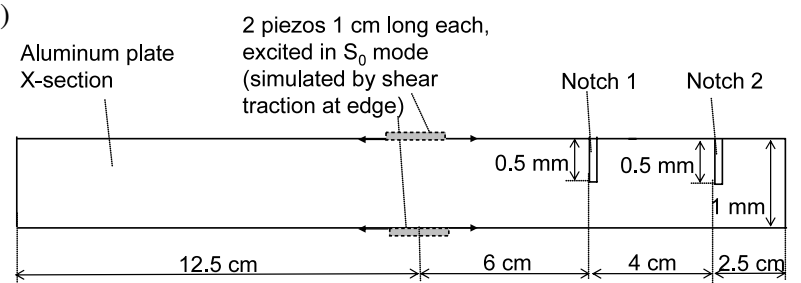

(c)

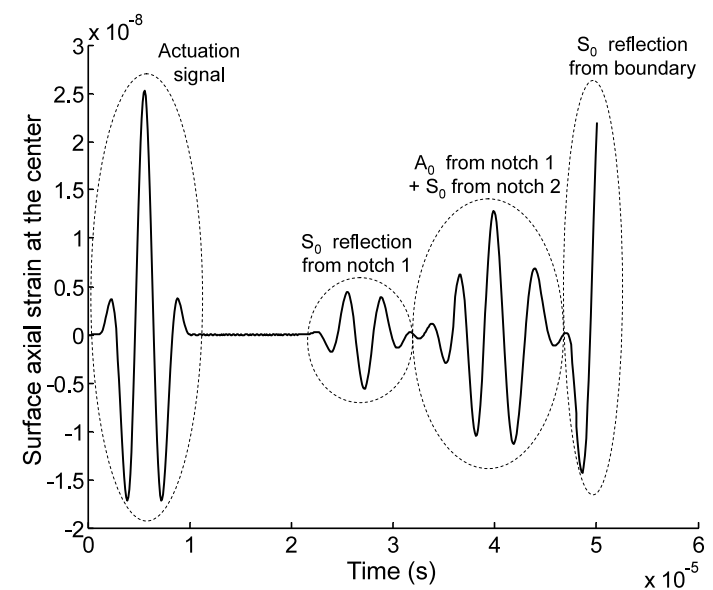

Figure 2. (a) 2D plate structure with one notch; (b) 2D plate structure with two notches; (c) surface axial strain waveform at the center for the structure in (b); (d) surface axial strain at the center for the structure in (a).

Lamb modes highlighted in figure 1 and the SH-modes) are possible in a $1 \mathrm{~mm}$ aluminum plate. Due to the $2 \mathrm{D}$ nature of the simulation, $\mathrm{SH}$ modes are not possible, and are therefore not considered. Thus, the two possible modes that can be reflected and transmitted from the defects are the $\mathrm{A}_{0}$ and $\mathrm{S}_{0}$ Lamb modes. The surface axial strain wave at the center of the plate from the FEM analysis, done using ABAQUS [9], is shown in figure 2(d). The first wave packet is the actuation pulse, which is followed by the $S_{0}$ mode reflection from the notch. Subsequently, the slower $\mathrm{A}_{0}$ mode reflection from the notch is received and finally the $S_{0}$ reflection from the boundary reaches the center of the structure. In this case, the presence and location of the notch was known beforehand, but in SHM, one has to estimate this information given the signal. The signal processing algorithm must decide what mode each reflected wave packet corresponds to, what the center frequency of the packet is (though the center frequency of excitation is known, the defect may be sensitive to higher or lower frequencies and therefore the center frequency of the reflection can change), and what the precise time of arrival is. Once the mode and the time-frequency center of the wave packet are known, the location of the defect can be estimated, knowing the group velocity for that mode. Now consider a similar structure as before (figure 2(b)), with the main difference being that there are two notches. In this case, as before, in the surface strain waveform at the center (figure 2(c)), one can see the actuation pulse, followed by the $S_{0}$ mode reflection from the notch closer to the center and the $\mathrm{S}_{0}$ mode boundary reflection. However, in this case, the $\mathrm{A}_{0}$ mode reflection from the notch closer to the center overlaps with the $S_{0}$ mode reflection from the notch closer to the free end. Therefore, the signal processing algorithm should also be able to separate overlapping multimodal reflections.
In addition, for SHM, since the signals are to be processed continuously in near-real-time, it is highly desirable to have a computationally efficient algorithm. Finally, the algorithm must be robust to noise.

\subsection{Objective of this work}

First, conventional solutions to the problem of GW signal processing for SHM are described and their shortcomings are highlighted. A new algorithm for $\mathrm{GW}$ signal processing for single point sensors using chirplet matching pursuits and mode correlation is then proposed and described in detail. The algorithm is designed for pulse-echo-based methodologies. Its ability to overcome the problems associated with conventional solutions for GW SHM is demonstrated using FEM and experimental results in isotropic plate structures. At the end, damage triangulation with multiple transducers in isotropic plate structures using this algorithm is discussed and demonstrated with experimental results.

\section{Conventional approaches to GW signal processing}

Conventional solutions to the problem of GW signal processing adapted from NDT are usually in the form of some time-frequency representation (TFR). Unlike the well-known Fourier transform, which provides 'global' information about the frequency content and is thereby suited for signals with stationary frequency content (meaning their frequency content does not change with time), TFRs yield the 'local' frequency content and are better suited for non-stationary-frequency signals. The simplest example of a TFR is the short time Fourier transform (STFT), in which the signal is divided into a number of small overlapping pieces in the time domain, each 

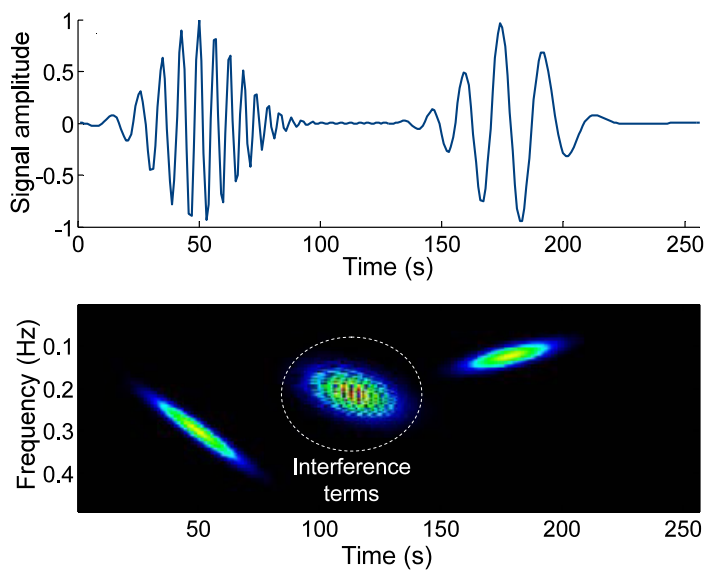

Figure 3. WVD of two linear modulated chirps.

piece is multiplied in time using a fixed modulation window and the Fourier transform is used on the resulting signal. Thus, the STFT, $S(t, \omega)$, of a signal, $s(t)$, and the corresponding time-frequency energy distribution, $E(t, \omega)$, obtained from it (called the spectrogram) are [10]

$$
\begin{gathered}
S(t, \omega)=\frac{1}{2 \pi} \int_{-\infty}^{\infty} s(\tau) h(\tau-t) \mathrm{e}^{-\mathrm{i} \omega \tau} \mathrm{d} \tau \\
E(t, \omega)=|S(t, \omega)|^{2}
\end{gathered}
$$

where $h(t)$ is the modulation window. Thus, an image is obtained for each point of the time-frequency plane $(t, \omega)$. This TFR can be implemented quickly using the fast Fourier transform (FFT) for digital signals. Another important TFR is the Wigner-Ville distribution (WVD), which is defined as [10]

$$
W(t, \omega)=\frac{1}{2 \pi} \int_{-\infty}^{\infty} s\left(t+\frac{\tau}{2}\right) s^{*}\left(t-\frac{\tau}{2}\right) \mathrm{e}^{-\mathrm{i} \omega \tau} \mathrm{d} \tau
$$

where $*$ indicates the complex conjugate. An advantage of the WVD is that it can exactly localize sinusoids, Dirac impulses and linear chirps. However, for other signals it always has additional interference terms. Figure 3 illustrates this point using the WVD of a signal composed of two Gaussian modulated linear chirps. The interference terms can be reduced by using a smoothing filter $f(t, \omega)$ in the time-frequency plane. This yields the generic smoothed WVD [10]:

$$
S W(t, \omega)=\int_{-\infty}^{\infty} \int_{-\infty}^{\infty} f(t-\tau, \omega-\varpi) W(\tau, \varpi) \mathrm{d} \tau \mathrm{d} \varpi .
$$

In fact, it can be shown [10] that the spectrogram and energy distribution from all commonly used TFRs such as the scalogram (which derives from the wavelet transform), the Hilbert Huang spectrum, and others can be represented in the form of equation (3). The disadvantage of smoothed WVDs is that they no longer can exactly localize linear chirps, sinusoids and Dirac impulses. One always compromises between the interference terms and time-frequency resolution. Further discussion on TFRs can be found in the books by Cohen [10] and Mallat [11]. For GW signal processing, researchers typically use some smoothed WVD followed by post-processing on the images. This isolates GW packets

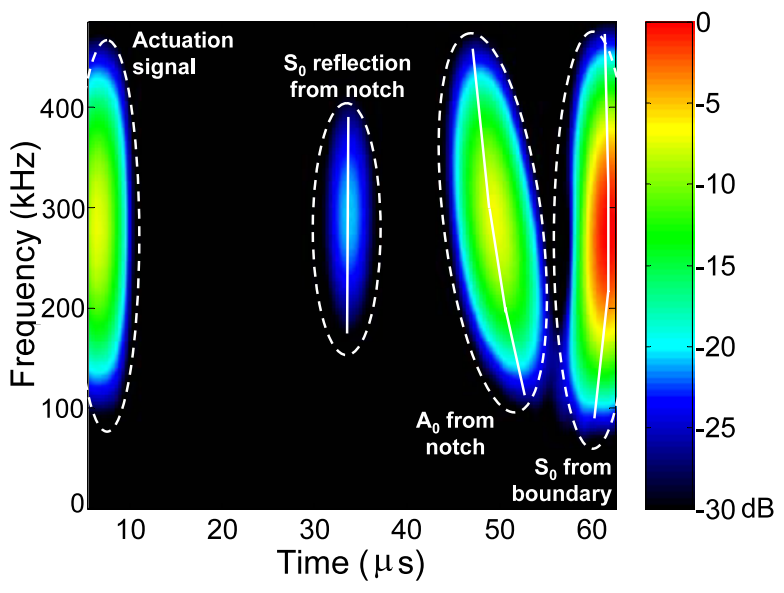

Figure 4. Spectrogram of signal in figure 2(d).

and locates their time-frequency centers, spread in the timefrequency plane and total energy. Finally, their modes are classified using the time-frequency 'ridges' of the reflections (these are the loci of the frequency centers for each time instant within each reflection). For example, Prosser et al [12] used the pseudo-WVD to process GW signals for material characterization of composites. Niethammer et al [13] reviewed four different time-frequency energy distributions to gauge their effectiveness in analyzing GW signals, namely, the reassigned spectrogram, the reassigned scalogram, the smoothed WVD and the Hilbert spectrum. Reassignment is a post-processing technique for improving resolution and decreasing spread in TFRs. While each technique was found to have its strengths and weaknesses, the reassigned spectrogram emerged as the best candidate for resolving multiple, closely spaced GW modes in terms of time and frequency. Kuttig et al [14] and Hong et al [15] used new TFRs based on different versions of the chirplet transform which has additional degrees of freedom (time shear and frequency shear) compared to the STFT. It enables superior resolution compared to conventional TFRs, but this comes at the cost of greater computational complexity. The above works were all mainly concerned with material characterization or offline NDT. Among works that have used TFRs for GW SHM, Sohn et al [16] and Lemistre and Balageas [17] used scalograms while Ihn and Chang [18] used spectrograms. Quek et al [19] and Salvino et al [20] used the Hilbert-Huang transform to process GW signals in plate structures for SHM.

As an illustrative example, the spectrogram for the signal in figure 2(b) over the excited bandwidth is shown in figure 4 (the modulation window used was identical to that for the excitation signal). The spectrogram is plotted on a decibel scale (logarithmic) with the peak value over the image as reference. For this simple example, the STFT seems capable of isolating the individual reflections, identifying their timefrequency centers and classifying their modes using the timefrequency ridges, which are highlighted with white lines in each reflection. However, as is shown in section 5, these are, in general, incapable of resolving overlapping multimodal reflections. Superior TFRs that might be capable of resolving such overlapped signals typically have a high computational cost associated with them. Another drawback of smoothed 
Guided-wave signal processing using chirplet matching pursuits and mode correlation for SHM

WVDs is difficult automated post-processing. In addition, these are more suited for broadband signals, while in GW SHM, usually narrow-band signals are used, in order to minimize signal spreading due to dispersion.

Another approach that has been tried for GW signal processing is the use of multi-element sensor arrays, such as in the work by Alleyne and Cawley [21] and El Youbi et al [22]. In this approach, the information about the spatial variation of the data over the sensing area of the array is used to decide the mode of each reflection. That is, a multi-dimensional Fourier transform is applied to the signals involving both time and spatial transformations. However, a large number of closely spaced transducers to avoid aliasing and sophisticated multichannel data capture and processing hardware are needed to implement this approach for GW SHM.

\section{Chirplet matching pursuits}

The matching pursuits approach to signal processing is a relatively new concept introduced by Mallat and Zhang [23]. Qian and Chen [24] developed a similar algorithm independently around the same time. This 'greedy' algorithm iteratively projects the signal onto a large and redundant dictionary of waveforms and chooses a waveform from that dictionary that is best adapted to approximate part of the waveform. To understand this algorithm, consider a complex valued signal $f_{1}(t)$ that belongs to the Hilbert space $L^{2}(R)$, where $R$ is the set of real numbers. Suppose this space is an inner product space with the inner product $\langle\cdot, \cdot\rangle$. Then, the following hold:

$$
\begin{aligned}
& \left\|f_{1}\right\|=\int_{-\infty}^{\infty}\left|f_{1}(t)\right|^{2} \mathrm{~d} t<\infty \\
& \left\langle f_{1}, f_{2}\right\rangle=\int_{-\infty}^{\infty} f_{1}(t) f_{2}^{*}(t) \mathrm{d} t
\end{aligned}
$$

where $f_{2}(t)$ also belongs to $L^{2}(R)$. The property of finiteness of the 2-norm, defined by the first expression in equation (4), also holds for $f_{2}(t)$. A dictionary $D$ of all possible expected wave structures, or 'atoms', is used, i.e. $D=\left\{k_{i}\right\}$, where $k_{i} \in L^{2}(R)$ and $\left\|k_{i}\right\|=1$. The 2-norm is also used as a metric of signal energy in this work. Then, the matching pursuit algorithm decomposes a signal $f(t) \in L^{2}(R)$ into $m$ atoms in the following iterative way (with $R^{0} f=f$ ):

(a) Choose the best atom in $D$ :

$$
k_{i_{m}}=\underset{k_{i} \in D}{\arg \max }\left|\left\langle R^{m-1} f, k_{i}\right\rangle\right| .
$$

(b) Compute the new residual after subtracting the component along the best atom chosen in (a):

$$
R^{m} f=R^{m-1} f-\left\langle R^{m-1} f, k_{i_{m}}\right\rangle k_{i_{m}} .
$$

Thus, it decomposes the signal into a linear expansion of waveforms chosen to match best the signal structure. Noise, in general, is uniformly distributed over the timefrequency plane. Since the matching pursuit algorithm looks for concentrated energy chunks in the time-frequency plane, it is inherently robust to noise. Due to this approach, which is distinct from conventional TFRs, the time-frequency centers, the spread in the time-frequency plane and the
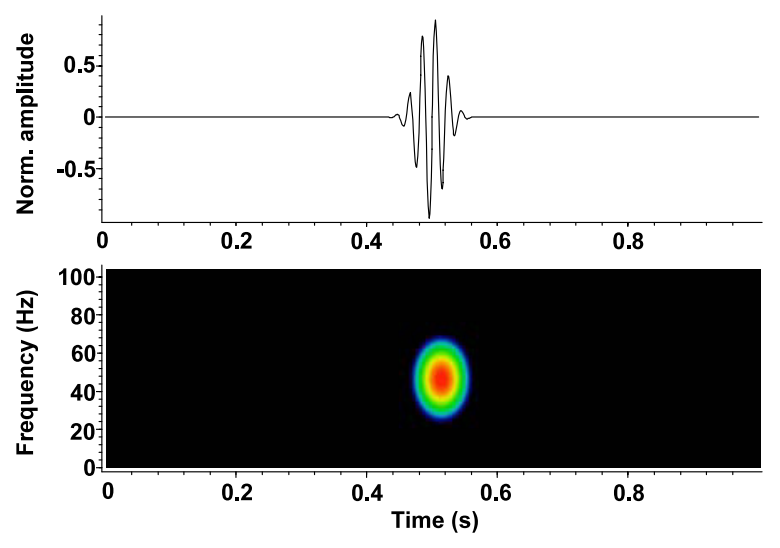

Figure 5. A stationary Gaussian atom and its WVD.

energy of the individual reflections are readily known, and no post-processing needs to be done on the output. It becomes much easier to automate this process in comparison to algorithms using conventional time-frequency representations. In those solutions, to automate the process, image processing algorithms would have to be used subsequent to the generation of the time-frequency plot to isolate the individual reflections.

In the original paper on matching pursuits [23], an efficient algorithm using a Gaussian modulated time-frequency atoms dictionary is described. This dictionary consists of the atoms:

$$
\begin{gathered}
k_{(l, u, \omega)}(t)=\frac{1}{\sqrt{l}} g\left(\frac{t-u}{l}\right) \exp (\mathrm{i} \omega(t-u)) \\
\text { with } g(t)=2^{1 / 4} \exp \left(-\pi t^{2}\right)
\end{gathered}
$$

where $u$ is the time center of the atom and $\omega$ is the angular frequency center of the atom. Also, $l$ is the scale of the atom, which is a metric representing the dilation along the time axis of the Gaussian window $g(t)$. It is indicative of the atom's time-frequency spread. These have stationary time-frequency behavior, i.e. the frequency at which the peak energy occurs for each time instant does not change with time, as would be seen in a WVD-like plot (see figure 5). Once the decomposition is done, it is possible to construct a time-frequency plot of the constituent atoms without the interference terms obtained using the conventional WVD. Thus, the resolution possible from such an approach is always superior to that from conventional smoothed WVDs. In addition, the use of Gaussian windows ensures that the atoms are optimal in terms of having minimal product for the rootmean-squared (RMS) pulse time width and RMS frequency bandwidth [25]. The matching pursuit algorithm with this dictionary has been explored by some researchers for GW signal analysis [26, 27]. However, the implicit assumption in these works is that the signals are unimodal and nondispersive. The atoms in this dictionary are ill-suited for analyzing dispersive signals, which have non-stationary timefrequency behavior. Furthermore, these atoms would not help in GW mode classification, since different modes with the same energy at the same time-frequency center would yield similar atoms.

More recently, Gribonval [28] introduced a computationally efficient algorithm for matching pursuits using a dictionary 


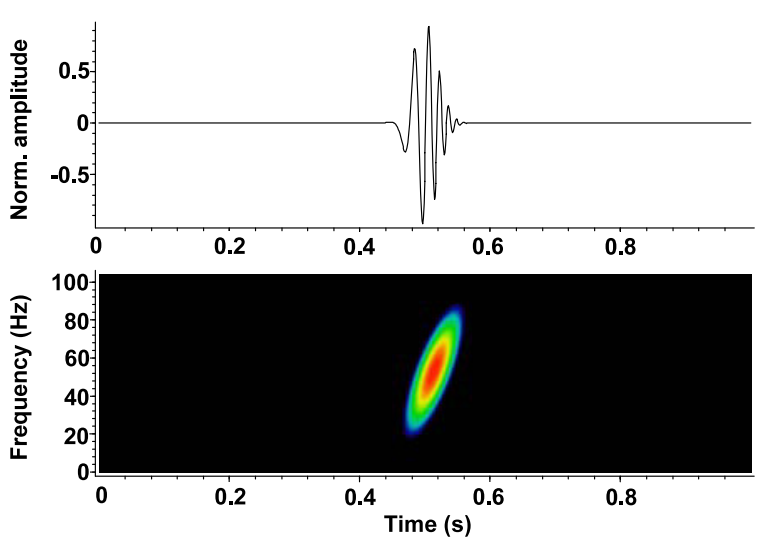

Figure 6. A Gaussian chirplet and its WVD.

consisting of Gaussian modulated chirplet atoms. That is, the dictionary comprises of atoms of the form:

$$
\begin{aligned}
& k_{(l, u, \omega, c)}(t) \\
& =\frac{1}{\sqrt{l}} g\left(\frac{t-u}{l}\right) \exp \left[\mathrm{i}\left(\omega(t-u)+\frac{c}{2}(t-u)^{2}\right)\right]
\end{aligned}
$$

where $c$ is the chirp rate of the atom. These have linear timefrequency behavior (see figure 6). Once the GW signal is decomposed into chirplets, the additional parameter, i.e. the chirp rate, can be used to identify the modes of the individual reflections. This algorithm is even more computationally efficient than the spectrogram. The computational time to decompose an $N$-point signal into $M$ atoms is just $\mathrm{O}(M N)$, whereas the complexity involved in generating the signal's spectrogram, not including post-processing, is $\mathrm{O}\left(N^{2} \log _{2} N\right)$. Thus, the chirplet matching pursuit seems a very attractive option for GW signal processing. In the next section, a detailed outline of the overall algorithm proposed using the chirplet matching pursuits approach is presented.

\section{Proposed algorithm for isotropic plate structures}

\subsection{Database creation}

This algorithm is designed for GW SHM in isotropic plate structures using the pulse-echo method. That is, the structure has a central actuator excited with a high frequency pulse and a collocated sensor receiving the GW echo pulses from the defects, if any, and the boundaries. This presumes that a set of baseline signals is available corresponding to the pristine condition for the structure. For this algorithm, initially a database of the chirplet chirp rates for the possible modes over the range of feasible time-frequency centers must be generated. The frequency centers are limited to the bandwidth excited in the structure, while the time centers are limited to the period between the end of the excitation signal and the time taken for the slowest mode from the boundary to reach the sensor. For this, it suffices to calculate these values for each mode at discrete points in the feasible region of the timefrequency plane. Then, use bilinear interpolation if values for other points are needed. It should be mentioned that in this work the scale $l$ of the chirplet atoms in the dictionary was kept fixed. The chosen value of $l$, say $l_{0}$, was such that the spread of the atom in the time domain was slightly larger than that of the excitation signal (20-30\% larger by rule of thumb; however, for very dispersive signals, this might need to be further increased). To generate the database, waveforms for each mode at the discrete time-frequency points are generated assuming the defect is a point-scatterer emitting circular crested waves. These waveforms represent the expected response of the piezoelectric wafer sensor collocated with the actuator. As discussed in earlier work by the authors [8], the response of a surface-bonded piezoelectric wafer is proportional to the average in-plane extensional strain over its surface area (this assumes that the sensor is thin and compliant enough to not affect the GW incident on it). For the FEM simulations, the waveforms represent the surface displacement along the plate thickness direction at the center of the plate. To do this, for each mode, the radial distance of the defect needs to be calculated. The phase velocity and group velocity curves for the isotropic plate structure are assumed known. Suppose the $\mathrm{S}_{0}$ mode was excited predominantly (or purely) and the excitation frequency is low enough so that the higher Lamb modes are not possible. Since a narrow bandwidth pulse is used, the group velocity can be used as the speed of pulse propagation to get defect location estimates. Therefore, the radial distance estimates for the possible modes at the timeangular frequency center $\left(t_{0}, \omega_{0}\right)$ are

$$
\begin{gathered}
r_{\mathrm{S}_{0}}=\frac{\left(t_{0}-t_{\mathrm{e}} / 2\right) \cdot c_{\mathrm{g}_{0}}\left(\omega_{0}\right)}{2} \\
r_{\mathrm{A}_{0}}=\frac{\left(t_{0}-t_{\mathrm{e}} / 2\right) \cdot c_{\mathrm{g}_{0}}\left(\omega_{0}\right) \cdot c_{\mathrm{g}_{\mathrm{A}_{0}}}\left(\omega_{0}\right)}{\left(c_{\mathrm{g}_{0}}\left(\omega_{0}\right)+c_{\mathrm{g}_{0}}\left(\omega_{0}\right)\right)}
\end{gathered}
$$

where $t_{\mathrm{e}}$ is the time span of the excitation signal and $c_{\mathrm{g}}\left(\omega_{0}\right)$ is the group velocity of a particular mode at angular frequency $\omega_{0}$. Furthermore, a minor correction term equal to half the actuator size along the direction of propagation is added to these estimates. This is because, for surface-bonded piezoelectric actuators, the GWs originate from the edge of the transducer, and not its center [8]. Next, the wavenumbers for each mode are calculated over the excited angular frequency range:

$$
\xi_{\mathrm{S}_{0}}(\omega)=\frac{\omega}{c_{\mathrm{ps}_{0}}(\omega)} \quad \xi_{\mathrm{A}_{0}}(\omega)=\frac{\omega}{c_{\mathrm{P}_{0}}(\omega)} .
$$

It is assumed that, after the GW excited by the actuator hits the defect, the defect becomes a point-source emitting circular crested waves axisymmetrically. The spatial variation of the piezoelectric sensor response is therefore described by the Hankel function of order zero [8]. Since this wave is reflected from the defect back towards the collocated actuator/sensor, it is an incoming wave. Therefore, if time dependence is of the form $\mathrm{e}^{\mathrm{i} \omega t}$, then the Hankel function of the first kind represents the incoming wave. For the case of symmetric mode reflection, the entire distance $2 r_{\mathrm{S}_{0}}$ is traversed as symmetric mode (since it was assumed that the $S_{0}$ mode was predominantly excited). For the case of antisymmetric mode reflection, half the total distance $2 r_{\mathrm{A}_{0}}$ (from the actuator to the defect) was traveled as $\mathrm{S}_{0}$ mode, whereas the second half was traveled as the $\mathrm{A}_{0}$ mode. Therefore, the harmonic surface strain response waveforms $Y(\omega)$ for the two cases are (ignoring constants of 
proportionality, since only the shape is of interest)

$$
\begin{gathered}
Y_{\mathrm{S}_{0}}(\omega)=H_{0}^{(1)}\left(\xi_{\mathrm{S}_{0}} \cdot r_{\mathrm{S}_{0}}\right) \cdot H_{0}^{(1)}\left(\xi_{\mathrm{S}_{0}} \cdot r_{\mathrm{S}_{0}}\right) \\
Y_{\mathrm{A}_{0}}(\omega)=H_{0}^{(1)}\left(\xi_{\mathrm{S}_{0}} \cdot r_{\mathrm{A}_{0}}\right) \cdot H_{0}^{(1)}\left(\xi_{\mathrm{A}_{0}} \cdot r_{\mathrm{A}_{0}}\right) .
\end{gathered}
$$

Here the effect of the piezosensor response being proportional to the average strain over its surface area is neglected for simplicity. For the 2D FEM simulations, the incoming wave is given by the complex exponential function with positive exponent:

$$
Y_{\mathrm{S}_{0}}=\mathrm{e}^{\mathrm{i} \xi_{0} r_{\mathrm{S}_{0}}} \cdot \mathrm{e}^{\mathrm{i} \xi \mathrm{s}_{0} r_{\mathrm{S}_{0}}}=\mathrm{e}^{\mathrm{i}\left(\xi \mathrm{s}_{0} 2 r_{\mathrm{S}_{0}}\right)} \quad Y_{\mathrm{A}_{0}}=\mathrm{e}^{\mathrm{i}\left(\xi \xi_{0} r_{\mathrm{A}_{0}}+\xi_{\mathrm{A}_{0}} r_{\mathrm{A}_{0}}\right)} .
$$

The chirplet matching pursuit scheme uses a database of Gaussian atoms. Therefore, to recover the time domain waveform $y(t)$ for a band-limited burst considering the frequency bandwidth and Gaussian modulation, the following equations are used:

$$
\begin{aligned}
y_{\mathrm{S}_{0}}(t) & =\int_{\omega_{0}-\Delta \omega / 2}^{\omega_{0}+\Delta \omega / 2} g\left(l_{0}\left(\omega-\omega_{0}\right)\right) \cdot\left(H_{0}^{(1)}\left(\xi_{\mathrm{S}_{0}} r_{\mathrm{S}_{0}}\right)\right)^{2} \mathrm{e}^{\mathrm{i} \omega t} \mathrm{~d} \omega \\
y_{\mathrm{A}_{0}}(t) & =\int_{\omega_{0}-\Delta \omega / 2}^{\omega_{0}+\Delta \omega / 2} g\left(l_{0}\left(\omega-\omega_{0}\right)\right) \cdot H_{0}^{(1)}\left(\xi_{\mathrm{S}_{0}} r_{\mathrm{A}_{0}}\right) \\
& \times H_{0}^{(1)}\left(\xi_{\mathrm{A}_{0}} r_{\mathrm{A}_{0}}\right) \mathrm{e}^{\mathrm{i} \omega t} \mathrm{~d} \omega
\end{aligned}
$$

where $\Delta \omega$ is the angular frequency bandwidth and $g()$ is the Gaussian window vector centered at angular frequency $\omega$ and with the chosen scale $l_{0}$. A similar equation holds for the 2D FEM simulations. Of course, in practice, this is implemented in the discrete (digital) domain. The inverse fast Fourier transform can be used for efficient computation.

It should be noted that, in this work, $\mathrm{SH}$ modes were not considered for the following reasons:

(a) In the FEM simulations, the elements were 2D, i.e. outof-plane displacements are not possible by design. Thus, $\mathrm{SH}$ modes are not possible.

(b) In the performed experiments, surface-bonded piezoelectric wafer transducers are used as sensors, which are almost entirely insensitive to shear waves. This is because they only sense the average in-plane surface extensional strain and not shear strain, as mentioned before.

Once these waveforms are generated, the chirplet matching algorithm is applied to them (restricting the scale of the dictionary chirplets to $l_{0}$ ) and the chirp rates corresponding to each mode at each point of the time-frequency grid are obtained. The chirplet matching pursuit was implemented using LastWave 2.0 [29], which is freeware. Thus, one has the database required to use the proposed algorithm for GW signal processing, which is described next.

\subsection{Processing the signal for damage detection and characterization}

The signal processing procedure consists of the following steps:

(i) The chirplet matching pursuit algorithm is applied to the difference between the test signal and the signal for the undamaged state. A dictionary of chirplets with fixed scale $s_{0}$ as discussed in section 4.1 is used. The algorithm is run until the last atom extracted has energy above a certain percentage of the first and most energetic extracted atom. In this work, this percentage was chosen to be $10 \%$. In the authors' experience, atoms below this threshold tend to correspond to approximation errors. This yields the timefrequency centers $\left(t_{0}, f_{0}\right)$, the chirp rates $(c)$ and the signal energies of the constituent atoms.

(ii) Atoms with frequency centers outside the excited bandwidth are neglected.

(iii) The most energetic atom in the time span not corresponding to the excitation signal or boundary reflections is examined. If it has energy above a certain threshold, the structure is judged damaged. There is no hard and fast rule to decide the value of this threshold, which is a critical parameter. The decision is dependent on the energy in the signal difference corresponding to the excitation time interval. In practice, no signal generator will be able to reproduce an excitation signal with $100 \%$ accuracy, and there is always some difference in the excitation signal as seen by the collocated sensor. In this work, the threshold was set to be $50 \%$ of the energy in the excitation signal difference. This might need to be lowered for structures with stronger damping characteristics. In addition, for the final SHM system, this threshold must also take into account false positive/false negative probabilities and risk assessment, which are highly application-dependent. Due to the impossibility of perfect reproduction of the excitation signal, there is a small blind zone in the vicinity of the collocated actuatorsensor pair. This is associated with the sensor being unable to distinguish the small-amplitude GW reflections from defects that might be very close to the actuator from the strong first transmitted pulse from the actuator.

(iv) Next, mode correlation is done using the atom's chirp rate. It is compared with that of the possible modes for the same time-frequency center in the database. The mode is identified as the one that minimizes the absolute value of the difference between the atom's chirp rate and the chirp rate for each mode at the same time-frequency center.

(v) Knowing the mode and time-frequency center of each atom, the defect's radial location relative to the transducer is known. The defect can then be characterized by using the frequency center, the energy in the reflection from the defect, and the relative modal contributions from the defect. This information can be used to infer what the defect type is in conjunction with an artificial neural network trained using prior experimental data or some modeling studies.

\section{Demonstration of the algorithm's capabilities}

\subsection{FEM simulations}

In section 1.3, it was seen that the STFT was capable of isolating the individual reflections and identifying their modes for the simple case of the GW signal in figure 2(d). Now consider the more complex signal in figure 2(c), with overlapping multimodal reflections. The portion of the signal between the end of the excitation signal and the start of 

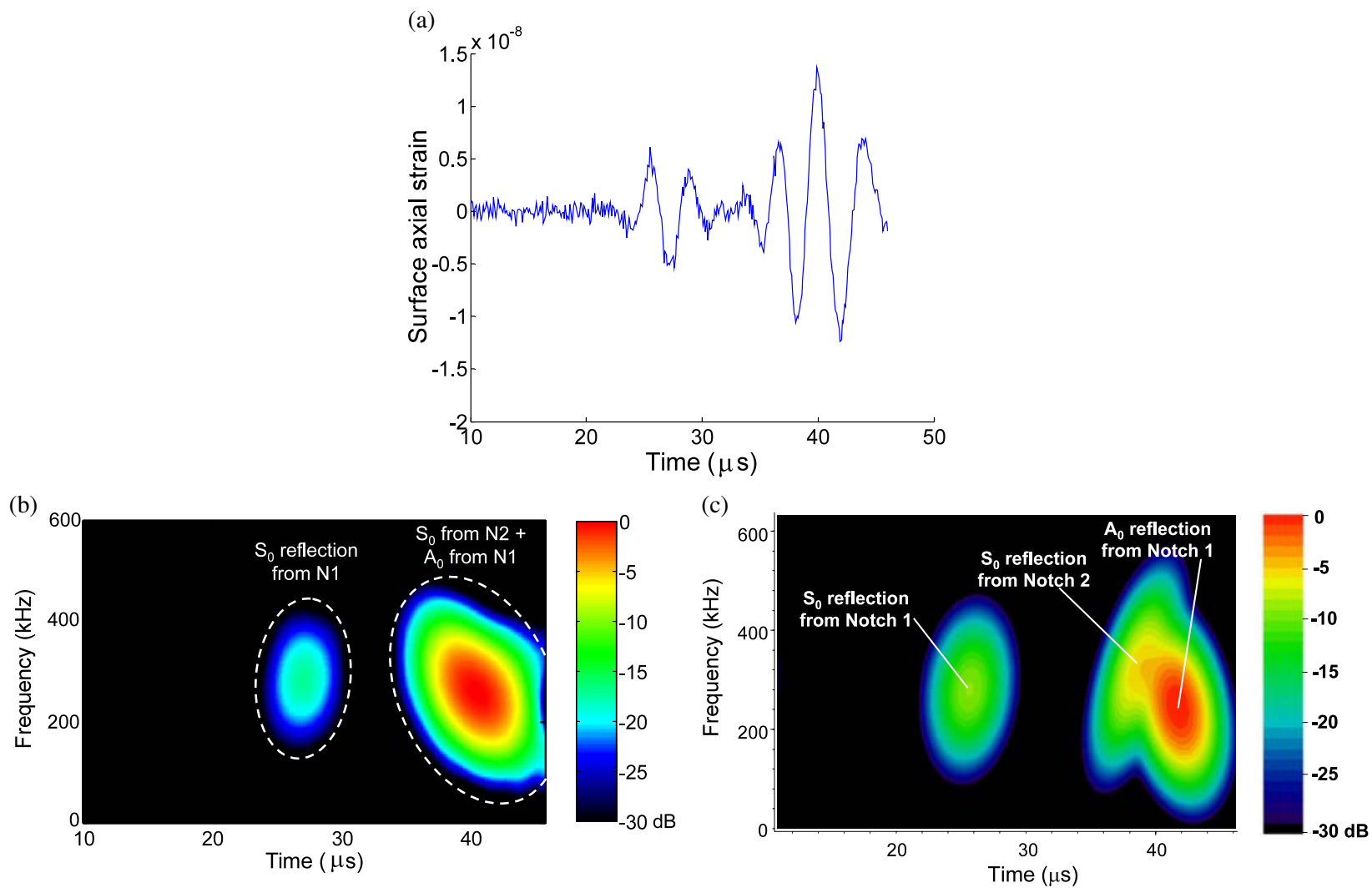

Figure 7. (a) Portion of signal in figure 2(c) with overlapping multimodal reflections and corrupted with artificial noise; (b) spectrogram of the signal in (a); (c) interference-free WVD of constituent chirplet atoms for the signal in (a).

Table 1. Simulated notch damage in FEM simulation (key: $c_{\mathrm{A}_{0}} \equiv$ chirp rate from database assuming $\mathrm{A}_{0}$ mode reflection; $c_{\mathrm{S}_{0}} \equiv$ chirp rate from database assuming $\mathrm{S}_{0}$ mode reflection; Mode $\equiv$ identified mode from mode correlation step; $r$ actual $\equiv$ actual radial location of notch; $r$ from new algo. $\equiv$ estimate of radial location of notch from proposed algorithm; $r$ from spect. $\equiv$ estimate of radial location of notch from spectrogram).

\begin{tabular}{llllllllrr}
\hline $\begin{array}{l}\text { Chirplet } \\
\text { no. }\end{array}$ & $\begin{array}{l}t_{0} \\
(\mu \mathrm{s})\end{array}$ & $\begin{array}{l}f_{o} \\
(\mathrm{kHz})\end{array}$ & $\begin{array}{l}c \\
\left(\mathrm{kHz} \mu \mathrm{s}^{-1}\right)\end{array}$ & $\begin{array}{l}\text { Signal energy } \\
(2-n o r m)\end{array}$ & $\begin{array}{l}c_{\mathrm{A}_{0}} \\
\left(\mathrm{kHz} \mu \mathrm{s}^{-1}\right)\end{array}$ & $\begin{array}{l}c_{\mathrm{S}_{0}} \\
\left(\mathrm{kHz} \mu \mathrm{s}^{-1}\right)\end{array}$ & $\begin{array}{l}r \text { actual } \\
(\mathrm{cm})\end{array}$ & $\begin{array}{l}r \text { from } \\
\text { new algo. }(\mathrm{cm})\end{array}$ & $\begin{array}{l}r \text { from } \\
\text { spect. }(\mathrm{cm})\end{array}$ \\
\hline 1 & 41.6 & 245.9 & -13 & $1.4 \times 10^{-14}$ & -12.5 & 0.6 & $\mathrm{~A}_{0}$ & 6.0 & 6.4 \\
2 & 38.4 & 319.6 & 34 & $3.7 \times 10^{-15}$ & -10.2 & 0.5 & $\mathrm{~S}_{0}$ & 10.0 & 9.4 \\
3 & 25.6 & 280.8 & 7.4 & $1.8 \times 10^{-15}$ & -7.9 & 0.3 & $\mathrm{~S}_{0}$ & 6.0 & 5.9 \\
\hline
\end{tabular}

the boundary reflection, after artificial corruption with white Gaussian noise (of amplitude $5 \%$ of the peak value in the signal), is shown in figure $7(a)$. The spectrogram for this signal (again, using a modulation window identical to that in the excitation signal) is shown in figure 7(b), on a decibel scale relative to the peak value in the image. The spectrogram cannot separate the overlapping multimodal reflections from the two notches, which are smeared together in the spectrogram. The time-frequency plot from the chirplet decomposition using the matching pursuit algorithm is shown in figure 7(c), also on a decibel scale. The power of this approach is evident from this figure, where clearly the individual overlapping reflections from the two notches are resolved. In addition, as highlighted in table 1 , the modes of the individual reflections are correctly identified and the axial locations of the notches are identified with a maximum deviation of $0.6 \mathrm{~cm}$, or $6 \%$ of the distance from the transducer. For the two reflections that the spectrogram could isolate, the errors for radial estimates are greater than that from the proposed algorithm. Thus, the proposed algorithm shows superior resolution compared to the spectrogram.

\subsection{Experimental results}

In order to verify the proposed algorithm's potential capabilities, experiments were conducted with a $1 \mathrm{~mm}$ thick aluminum plate structure, the schematic of which is shown in figure 8 (a). The $1 \mathrm{~mm}$ thick aircraft-grade aluminum alloy plate was supported on two support struts on two edges and the other two edges were free. Surface-bonded PZT-5A piezoceramic transducers were used. The actuators were excited symmetrically with a 2.5 -cycle Hann-windowed sinusoidal toneburst of center frequency $175 \mathrm{kHz}$, thereby predominantly exciting the $S_{0}$ mode. After baseline signals were recorded for the pristine condition, artificial 'damage' sites in the form of C-clamps were introduced (see figure 8(b)). The C-clamps act as local scatterers of GWs incident on them 
(a)

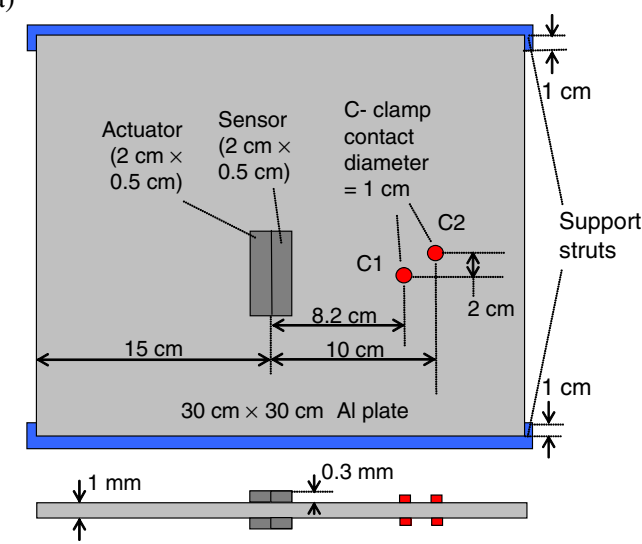

(b)

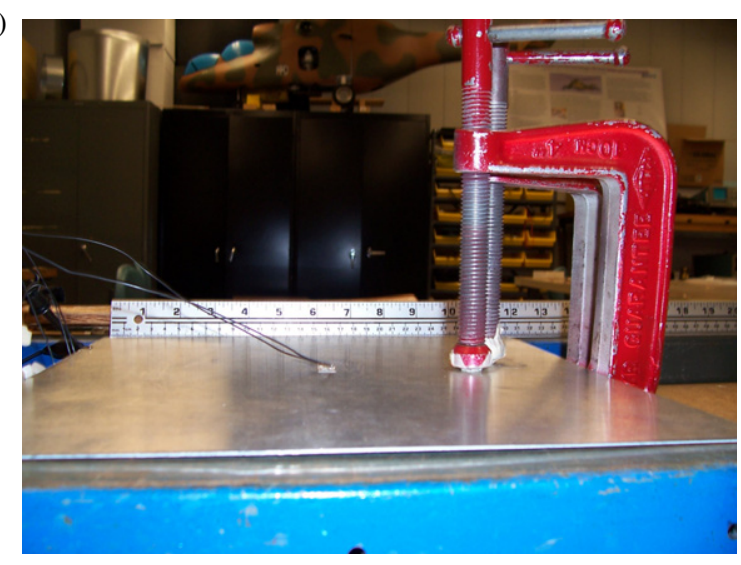

Figure 8. (a) Schematic of experimental set-up; (b) photograph of experimental set-up.

over their contact area, causing incident GWs to be scattered from them. Damage in the structure, such as cracks, dents or impact damage, would also have a similar effect on GWs incident on it. The difference signal between the pristine and 'damaged' cases is shown in figure 9(a). Again, in this case, the spectrogram is incapable of resolving the overlapping $\mathrm{S}_{0}$ mode reflections from the two clamps (figure $9(\mathrm{~b})$ ). On the other hand, the proposed algorithm showed its superior resolution in this case too. The chirplet matching pursuit step was able to resolve the overlapping $S_{0}$ mode reflections as well as the $\mathrm{S}_{0}$ and $\mathrm{A}_{0}$ mode reflections from the boundary (figure 9(c)). The mode correlation step correctly identified the modes, thereby allowing accurate radial location estimates of the clamps, as seen in table 2 (errors in location: $\mathrm{C} 1-0.3 \mathrm{~cm}$; $\mathrm{C} 2-0.9 \mathrm{~cm}$ ). The spectrogram's estimated location (for the reflection from the clamp that it could localize) has the same error as the proposed algorithm. When using the relative modal contributions to characterize the defect, one must bear in mind that a finite-dimensional piezoceramic sensor has different sensitivities to different wavelengths of the GW sensed [8]. As a first-order approximation, it might suffice to normalize the energy of each reflection to the sensitivity of the sensor to the wavelength corresponding to the center frequency for the GW mode of the reflection.

It should be noted that the best accuracy in radial location estimation was in the FEM simulation with the $\mathrm{S}_{0}$ mode reflection from $\mathrm{N} 1$ (error: $0.1 \mathrm{~cm}$ ). There are two reasons for this: (i) the reflection was isolated (i.e. not overlapping with another reflection) and (ii) the notch was very thin axially $(0.025 \mathrm{~cm})$, and hence the 'point-scatterer' defect model was realistic. In the experiment, the clamp had a contact diameter of $1 \mathrm{~cm}$, weakening this assumption, as reflected in the location errors. Furthermore, the error tends to be worse for the weaker reflection in overlapping reflections, as one would naturally expect. Another error source is the uncertainty in material properties, which affects wavespeeds. Despite these errors, which are minor, the advantages of the new algorithm over conventional approaches to $\mathrm{GW}$ signal processing can be clearly seen with these results. However, it should be pointed out that testing was restricted to the fundamental GW modes in this work. At higher frequencies, in the presence of higher GW modes, the use of linear chirplets may not suffice. Quadratic or higher-order chirplets might need to be employed, such as in the work by Hong et al [30]. In that work, the matching pursuit approach was used with quadratic chirp functions for GW signal processing. However, this will increase the computational complexity of the algorithm compared to that for linear chirplets. In addition, in that work, sensing was restricted to one mode there (by controlling the number of coil turns in the magnetostrictive GW sensor used) and mode classification was not addressed. It should be noted that, in this work, the two modes had different dispersion characteristics over the excited frequency bandwidth. If the two modes are similar to each other in terms of variation of wavespeed with frequency, the chirp rates for the two modes may be very close to each other. The algorithm presented here may not be able to distinguish the modes. Until this point, only radial location of defects relative to a transducer pair has been discussed. In the next section, triangulation using multiple transducers in isotropic plates is discussed.

\section{Triangulation in isotropic plate structures}

In order to pinpoint the in-plane location of a defect in an isotropic plate structure and characterize it, one needs the radial locations of the defect relative to at least three pairs of central collocated piezoelectric transducers. It is highly desirable to use circular or ring-shaped transducer wafers, so that there is no directional selectivity or preference. In addition, care must be taken to use as thin piezoelectric wafers as possible to minimize the extraneous reflections caused by the increased local stiffness of the structure where the transducer is bonded. The proposed algorithm needs to be repeated for the signals obtained using each collocated actuator/sensor pair. If there are multiple mode reflections observed from the defect, the average radial location obtained from the modes can be used. One can then draw three circles of radii equal to the radial locations thus found about the centers of the corresponding actuator/sensor pairs. The intersection of the three circles would yield the location of the defect. This is illustrated in figure 10(a). In addition, as before, the relative modal contributions, the frequency center and individual modal energies can be as input parameters for a pattern recognition algorithm used to classify the defect and quantify its severity. 

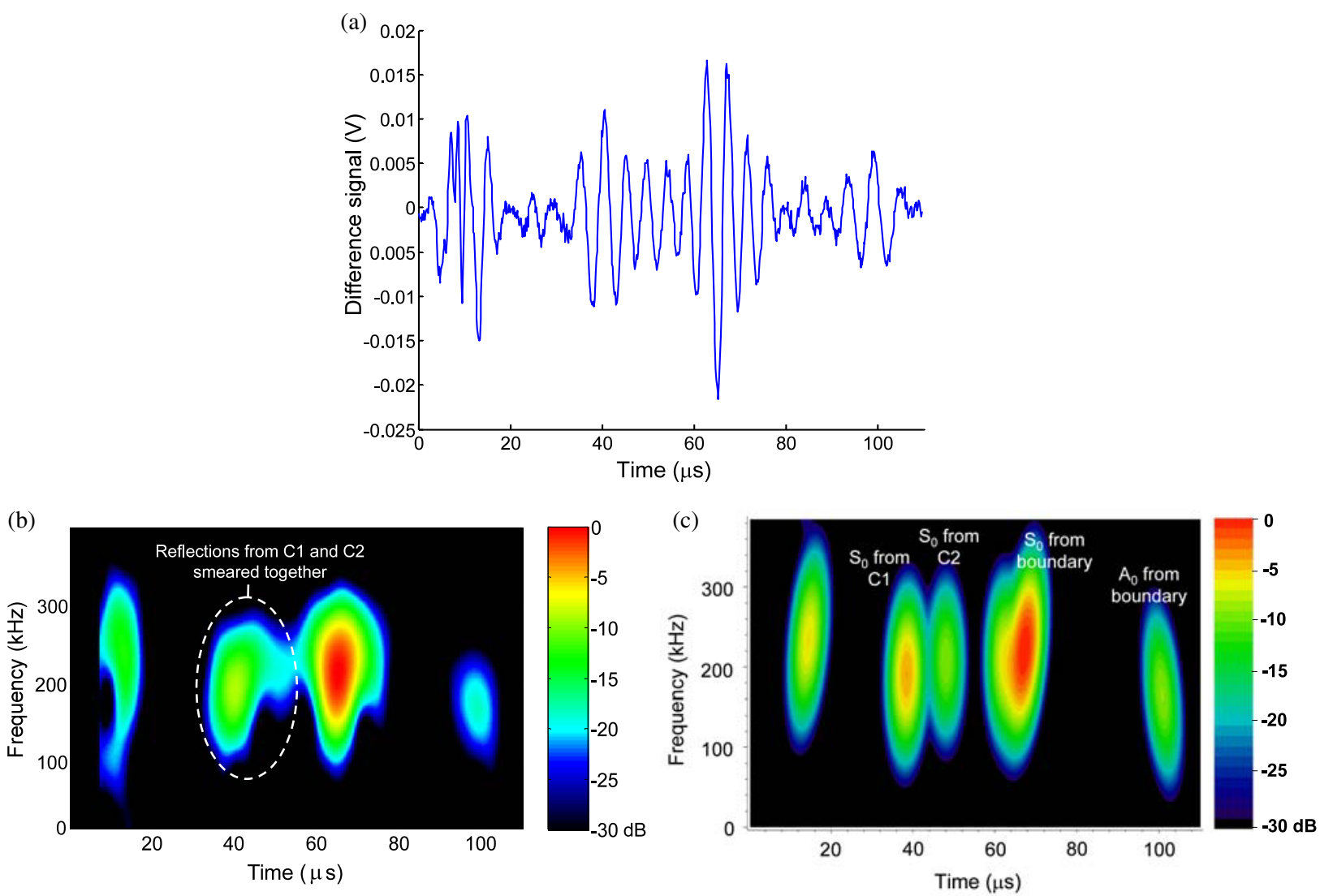

Figure 9. (a) Difference signal between pristine and 'damaged' states; (b) spectrogram of the signal in (a); (c) interference-free WVD of constituent chirplet atoms for the signal in (a).

Table 2. Experimental results of isotropic plate with simulated damage (key: $c_{\mathrm{A}_{0}} \equiv$ chirp rate from database assuming $\mathrm{A}_{0}$ mode reflection $c_{\mathrm{S}_{0}} \equiv$ chirp rate from database assuming $\mathrm{S}_{0}$ mode reflection; Mode $\equiv$ identified mode from mode correlation step; $r$ actual $\equiv$ actual radial location of clamp; $r$ from new algo. $\equiv$ estimate of radial location of clamp from proposed algorithm; $r$ from spect. $\equiv$ estimate of radial location of clamp from spectrogram; Bndry $\equiv$ boundary reflection; Exctn signal $\equiv$ difference in excitation signal).

\begin{tabular}{|c|c|c|c|c|c|c|c|c|c|c|}
\hline $\begin{array}{l}\text { Chirplet } \\
\text { no. }\end{array}$ & $\begin{array}{l}t_{0} \\
(\mu \mathrm{s})\end{array}$ & $\begin{array}{l}f_{o} \\
(\mathrm{kHz})\end{array}$ & $\begin{array}{l}c \\
\left(\mathrm{kHz} \mu \mathrm{s}^{-1}\right)\end{array}$ & $\begin{array}{l}\text { Signal energy } \\
\text { (2-norm) }\end{array}$ & $\begin{array}{l}c_{\mathrm{A}_{0}} \\
\left(\mathrm{kHz} \mu \mathrm{s}^{-1}\right)\end{array}$ & $\begin{array}{l}c_{\mathrm{S}_{0}} \\
\left(\mathrm{kHz} \mu \mathrm{s}^{-1}\right)\end{array}$ & Mode & $\begin{array}{l}r \text { actual } \\
(\mathrm{cm})\end{array}$ & $\begin{array}{l}r \text { from } \\
\text { new algo. }(\mathrm{cm})\end{array}$ & $\begin{array}{l}r \text { from } \\
\text { spect. }(\mathrm{cm})\end{array}$ \\
\hline 1 & 67.2 & 221.5 & 7.8 & $7.3 \times 10^{-3}$ & -5.11 & 0.14 & $\mathrm{~S}_{0}$ & Bndry & Bndry & Bndry \\
\hline 2 & 38.4 & 190.9 & 1.7 & $2.9 \times 10^{-3}$ & -4.55 & 0.07 & $\mathrm{~S}_{0}$ & 8.2 & 8.5 & 8.5 \\
\hline 3 & 14.4 & 234.2 & 7.7 & $1.9 \times 10^{-3}$ & -1.79 & 0.01 & Exctn signal & & & \\
\hline 4 & 62.4 & 208.3 & 0.0 & $1.8 \times 10^{-3}$ & -5.17 & 0.13 & $\mathrm{~S}_{0}$ & Bndry & Bndry & Bndry \\
\hline 5 & 100.8 & 166.1 & -7.6 & $9.4 \times 10^{-4}$ & -4.63 & 0.33 & $\mathrm{~A}_{0}$ & Bndry & Bndry & Bndry \\
\hline 6 & 48.0 & 208.3 & 0.0 & $9.2 \times 10^{-4}$ & -4.76 & 0.09 & $\mathrm{~S}_{0}$ & 10.2 & 11.1 & NA \\
\hline
\end{tabular}

If, however, one is mainly interested in locating the defect and not in characterizing it, an easier approach can be adopted. Instead of using three collocated piezoelectric actuator/sensor pairs, it suffices to use three circular piezoelectric wafer transducers, and while one is excited, the others can be used as sensors. However, in this approach, one must ensure that the elements are sensitive only to one mode when used as sensors. Then, the chirplet matching pursuit step is used to find the time-of-flight from the actuator to the defect and back to one of the sensors as well as the frequency center of the pulse. This yields the distance traveled by the pulse, say $d$ (since only one group velocity is possible). The locus of all possible locations of the defect is an ellipse with the actuator and the sensor as its foci and $d$ as the major axis. By exciting each actuator in turn and using the others as sensors, three such ellipses can be drawn and the defect is located at their intersection. This concept was proven experimentally using a $3.15 \mathrm{~mm}$ thick aluminum 5052 alloy plate instrumented with three surface-bonded piezoelectric discs of diameter $1.3 \mathrm{~cm}$ each and thickness $0.23 \mathrm{~mm}$ each. The excitation signal used was a 2.5-cycle Hann windowed sinusoidal toneburst with center frequency $210 \mathrm{kHz}$. At this frequency and in its vicinity, the $\mathrm{A}_{0}$ mode wavelength nearly equals the transducer diameter. Therefore, as proved in an earlier modeling paper by the authors [8], the transducers are insensitive to $\mathrm{A}_{0}$ modes when used as sensors, and only the $S_{0}$ mode needs to be considered. The results from this experiment are shown in figure 10(b). A through-hole of diameter $5 \mathrm{~mm}$ was drilled into the plate as shown to check if its location could be found using this approach. While one expects the three ellipses to 
(a)

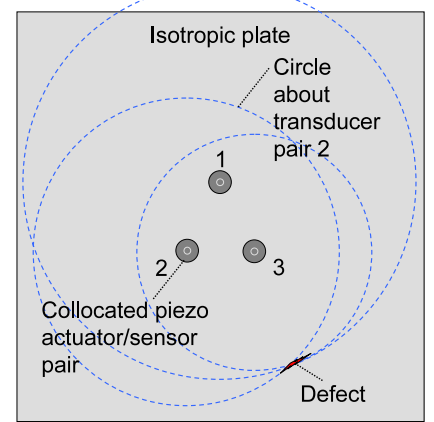

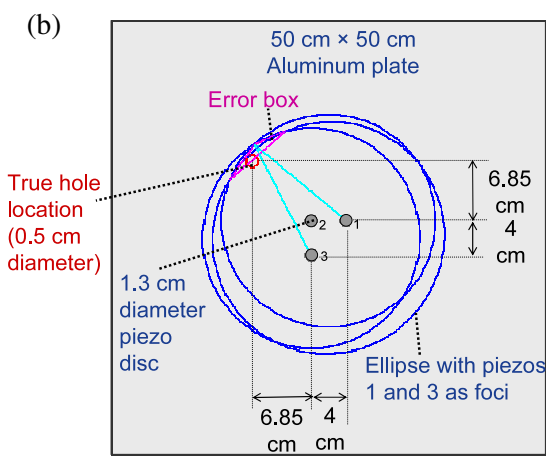

Figure 10. (a) Approach for locating and characterizing defects in the plane of plate structures using multimodal signals; (b) experimental results for in-plane defect location in plate structures using unimodal GW signals.

intersect at one point, due to experimental imperfections, they come close to intersecting each other at a single point but do not quite do so, resulting in a triangular error box. This gives a crude estimate of the defect size. The center of the error box was $0.5 \mathrm{~cm}$ away from the center of the drilled hole. It should be noted that in this simplified approach, it is crucial to restrict the sensing to one mode. If more than one mode is possible, the locus of all points of the defect given the timeof-flight and center frequency from one transducer to another is not necessarily an ellipse. Since one cannot be sure about how much of the time was spent traveling as one mode and how much as another, the locus would, in general, be an intricate shape and this shape would need to be recalculated for different times of flight, thereby making the algorithm computationally intensive. This ellipse triangulation technique has been discussed in the open literature (e.g. Kehlenbach and Das [31]), but the case of multimodal signals has not received much attention.

\section{Summary and future directions}

This paper presented a new approach for GW signal processing using chirplet matching pursuits and mode correlation. The algorithm is designed for processing GW signals obtained in pulse-echo tests for SHM. Its theoretical advantages over conventional algorithms for GW SHM were discussed: better resolution and lack of interference terms (enables it to separate overlapping multimodal reflections), robustness to noise, computational efficiency (in this aspect, it is even superior to the spectrogram), and ease-to-automate postprocessing, as needed for SHM. Its prowess was demonstrated using numerical and experimental results, where the proposed algorithm was able to separate overlapping, multimodal reflections and estimate radial locations of defects with a maximum deviation of $0.9 \mathrm{~cm}$ for a damage site $10.2 \mathrm{~cm}$ away. The resolution of the algorithm was shown to be superior or equal to that using a spectrogram. In-plane triangulation for isotropic plate structures using multimodal signals and ellipse triangulation using unimodal GW signals were discussed. The latter was experimentally demonstrated to triangulate a drilled hole within one diameter of it in an aluminum alloy plate. Future work will focus on experimental validation of an extension of the algorithm for composite structures. In addition, detailed models for GW scattering from defects will be examined to move beyond using the 'point-scatterer' assumption used for defects in this work.

\section{Acknowledgments}

The authors gratefully acknowledge the help of Ms Monika Patel (University of Michigan) with the set-up for the triangulation experiment. This work was supported by the Space Vehicle Technology Institute under grant NCC3-989 jointly funded by NASA and DOD within the NASA Constellation University Institutes Project, with Ms Claudia Meyer as the project manager.

\section{References}

[1] NTSB Aviation Accident Report DCA88MA054 1988 (Washington, DC: National Transportation Safety Board)

[2] NASA Columbia Accident Investigation Board Report 2003 (Washington, DC: Government Printing Office)

[3] NTSB Aviation Accident Report DCA06MA010 2005 (Washington, DC: National Transportation Safety Board)

[4] Farrar C R, Lieven N A J and Bement M T 2005 An introduction to damage prognosis Damage Prognosis ed D Inman, C R Farrar, V Lopes Jr and V Steffen $\mathrm{Jr}$ (Chichester: Wiley)

[5] Raghavan A and Cesnik C E S 2005 Lamb-wave based structural health monitoring Damage Prognosis ed D Inman, C R Farrar, V Lopes Jr and V Steffen Jr (Chichester: Wiley)

[6] Miklowitz J 1978 The Theory of Elastic Waves and Waveguides (New York: North-Holland)

[7] Graff K F 1991 Wave Motion in Elastic Solids (New York: Dover)

[8] Raghavan A and Cesnik C E S 2005 Finite dimensional piezoelectric transducer modeling for guided wave based structural health monitoring Smart Mater. Struct. 14 1448-61

[9] ABAQUS/Standard User's Manual Version 6.22001 (Providence, RI: Hibbitt, Karlsson and Sorensen)

[10] Cohen L 1995 Time-Frequency Analysis (Englewood Cliffs, NJ: Prentice-Hall)

[11] Mallat S 1999 A Wavelet Tour of Signal Processing (San Diego, CA: Academic)

[12] Prosser W H, Seale M D and Smith B T 1999 Time-frequency analysis of the dispersion of Lamb modes J. Acoust. Soc. Am. 105 2669-76

[13] Niethammer M, Jacobs L J, Qu J and Jarzynski J 2001 Time-frequency representations of Lamb waves J. Acoust. Soc. Am. 109 1841-7

[14] Kuttig H, Niethammer M, Hurlebaus S and Jacobs L J 2006 Model-based analysis of dispersion curves using chirplets J. Acoust. Soc. Am. 119 2122-30 
[15] Hong J-C, Sun K H and Kim Y Y 2005 Dispersion-based short-time Fourier transform applied to dispersive wave analysis J. Acoust. Soc. Am. 117 2949-60

[16] Sohn H, Park G, Wait J R, Limback N P and Farrar C R 2004 Wavelet based active sensing for delamination detection in composite structures Smart Mater. Struct. 13 153-60

[17] Lemistre M and Balageas D 2001 Structural health monitoring system based on diffracted Lamb wave analysis by multiresolution analysis Smart Mater. Struct. 10 504-11

[18] Ihn J-B and Chang F-K 2004 Detection and monitoring of hidden fatigue crack growth using a built-in piezoelectric sensor/actuator network: I. Diagnostics Smart Mater. Struct. 13 609-20

[19] Quek S T, Tua P S and Wang Q 2003 Detecting anomalies in beams and plate based on the Hilbert-Huang transform of real signals Smart Mater. Struct. 12 447-60

[20] Salvino L, Purekar A and Pines D 2005 Health monitoring of 2D plates using empirical mode decomposition and Hilbert phase Proc. 4th Int. Workshop on Structural Health Monitoring (Stanford University, CA, Sept. 2005)

[21] Alleyne D and Cawley P 1991 A two-dimensional Fourier transform method for the measurement of propagating multimode signals J. Acoust. Soc. Am. 89 1159-68

[22] El Youbi F, Grondel S and Assaad J 2004 Signal processing for damage detection using two different array transducers Ultrasonics 42 803-6
[23] Mallat S and Zhang Z 1993 Matching pursuits with time-frequency dictionaries IEEE Trans. Signal Process. 41 3397-415

[24] Qian S and Chen D 1994 Signal representation via adaptive normalized Gaussian functions Signal Process. 36 1-11

[25] Bracewell R 2000 The Fourier Transform and its Applications 3rd edn (New York: McGraw-Hill)

[26] Zhang G, Zhang S and Wang Y 2000 Application of adaptive time-frequency decomposition in ultrasonic NDE of highly scattering materials Ultrasonics 38 961-4

[27] Hong J-C, Sun K H and Kim Y Y 2005 The matching pursuit approach based on the modulated Gaussian pulse for efficient guided wave inspection Smart Mater. Struct. $14548-60$

[28] Gribonval R 2001 Fast matching pursuit with a multiscale dictionary of Gaussian chirps IEEE Trans. Signal Process. 49 994-1001

[29] Bacry E, LastWave 2.0 software, http://www.cmap. polytechnique. fr/ $\sim$ bacry/LastWave

[30] Hong J-C, Sun K H and Kim Y Y 2006 Waveguide damage detection by the matching pursuit approach employing the dispersion-based chirp functions IEEE Trans. Ultrason. Ferroelectr. Freq. Control 53 592-605

[31] Kehlenbach M and Das S 2002 Identifying damage in plates by analyzing Lamb wave propagation characteristics Proc. SPIE 4702 364-75 